RACAR : Revue d'art canadienne

Canadian Art Review

\title{
Association d'art des universités du Canada
}

Rapport de la réunion annuelle tenue à Montréal du 25 au 28

février 1981

\section{Universities Art Association of Canada}

\section{Claude Bergeron}

Volume 8, numéro 2, 1981

URI : https://id.erudit.org/iderudit/1075005ar

DOI : https://doi.org/10.7202/1075005ar

Aller au sommaire du numéro

\section{Éditeur(s)}

UAAC-AAUC (University Art Association of Canada | Association d'art des universités du Canada)

\section{ISSN}

0315-9906 (imprimé)

1918-4778 (numérique)

Découvrir la revue

\section{Citer ce compte rendu}

Bergeron, C. (1981). Compte rendu de [Association d'art des universités du Canada : rapport de la réunion annuelle tenue à Montréal du 25 au 28 février 1981]. RACAR : Revue d'art canadienne / Canadian Art Review, 8(2), 153-156. https://doi.org/10.7202/1075005ar

Tous droits réservés (C) UAAC-AAUC (University Art Association of Canada | Association d'art des universités du Canada), 1987
Ce document est protégé par la loi sur le droit d'auteur. L'utilisation des services d'Érudit (y compris la reproduction) est assujettie à sa politique d'utilisation que vous pouvez consulter en ligne.

https://apropos.erudit.org/fr/usagers/politique-dutilisation/ 


\title{
Association d'art des universités du Canada Universities Art Association of Canada
}

\author{
Rapport de la réunion annuelle tenue à Montréal \\ du 25 au 28 février 1981
}

Le congrès de 1981 de l'Association d'art des universités du Canada s'est tenu à l'Université du Québec à Montréal conjointement avec celui de l'Association internationale des critiques d'art / International Association of Art Critics. Le rapport qui suit a été rédigé grâce à la collaboration de quelques présidents de séances. Nous regrettons toutefois de n'avoir pu résumer les communications présentées aux séances suivantes : Discours au studio; Les arts des peuples aborigènes; Michael Snow et ses critiques; Les femmes artistes parlent d'elles-mêmes; L'engagement du critique d'art.

Perspectives historiques de la critique d'art MONIQUE. BRLNeT-WFinmanis, Université du Québec à Montréal, présidente de séance

Gerald Needham de l'Université York a soutenu que les critiques d'art les plus perspicaces du Xix ${ }^{\mathrm{e}}$ siècle ont tous reconnu l'importance de la gravure. Certains, comme Baudelaire, y voyaient le médium par excellence pour traiter de la vie moderne, tandis que d'autres, tels les Goncourt et Philippc Burty, s'adonnaient eux-mêmes à la pratique de la gravure.

Philippe Burty fut précisément le sujet de l'exposé de Monique Brunet-Weinmann qui, en plus de rappeler l'intérêt de celui-ci pour la gravure et son rôle de diffuseur de l'cstampe japonaise à une date très hâtive, souligna l'appui continu qu'il apporta au mouvement impressionniste.

Alors que (jilles Daigneault, critique d'art, s'est plaint de ce que les critiques négligent la gravure québécoise et, encore plus, les graveurs, Louise Letocha, directrice du Musée d'art contemporain dc Montréal, s'est appliquée à montrer la place de la fonction critique au scin d'un musée d'art contemporain.

L'artiste en tant que critique / Le critique en tant qu'artiste LAURENT IAMY, président de séance

Dans le cadre de cette table ronde, Vojtěch JiratWasiutynski de l'Université Qucen's a examiné les concepts de l'équivalence visuelle, de l'abstraction et de la nature "musicale" du symbolisme visuel que Gauguin, ainsi que Van (oogh et Émile Bernard avec qui il correspondait, a utilisé dans la genèse d'une peinture symboliste. Ces concepts de même que l'analogie du symbole religieux ont ensuite été repris par les Nabis avec qui Gauguin avait des contacts.

L'art médiéval WARRIN SANDERSON, Université Concordia, président de séance

Josef Polzer de l'Université Queen's a avancé l'hypothèse que les Méditations sur la vie de Jésus-Christ ont été inspirécs par un objectif de sécularisation de la dévotion religieuse à la fin du Moyen Âge.

Sarah M. McKinnon de l'Université de Winnipeg a étudić plusieurs enluminures de la fin du Moyen Âge représentant sainte Barbe et elle s'appliqua surtout à définir des rapports entre les tours, symboles de la sainte, qui y sont illustrées et l'architecture de l'époque.

Contrairement à l'opinion généralement admise selon layuelle la nef de la Temple Church de Londres serait postérieure au chour de Canterbury et qu'elle en dériverait, Malcolm Thurlby de l'Université Concordia, s'appuyant sur des considérations stylistiques, des dessins d'archéologues et des documents, conclut que l'ćglise fut commencée en 1161 et achevée avant 1181 . Elle ressort alors comme un édifice novateur qui a pu influencer Guillaume de Sens à Canterbury.

Étudiant la Nativité du pontifical du $x^{e}$ siècle fait pour l'évêque Ethelwold de Winchester, Robert Deshman de l'Université de Toronto explique que la crècheautel, pcinte comme un édifice et placée dans le coin inférieur de l'image, illustre que le Christ, par son incarnation, est la pierre angulaire qui a réuni les murs des Gentils et des Juifs, représentés par le bœuf et l'âne, dans l'édifice vivant de l'Église. Cette interprétation lui est suggérée par le texte du pontifical et par deux antiphonaires anglais. 


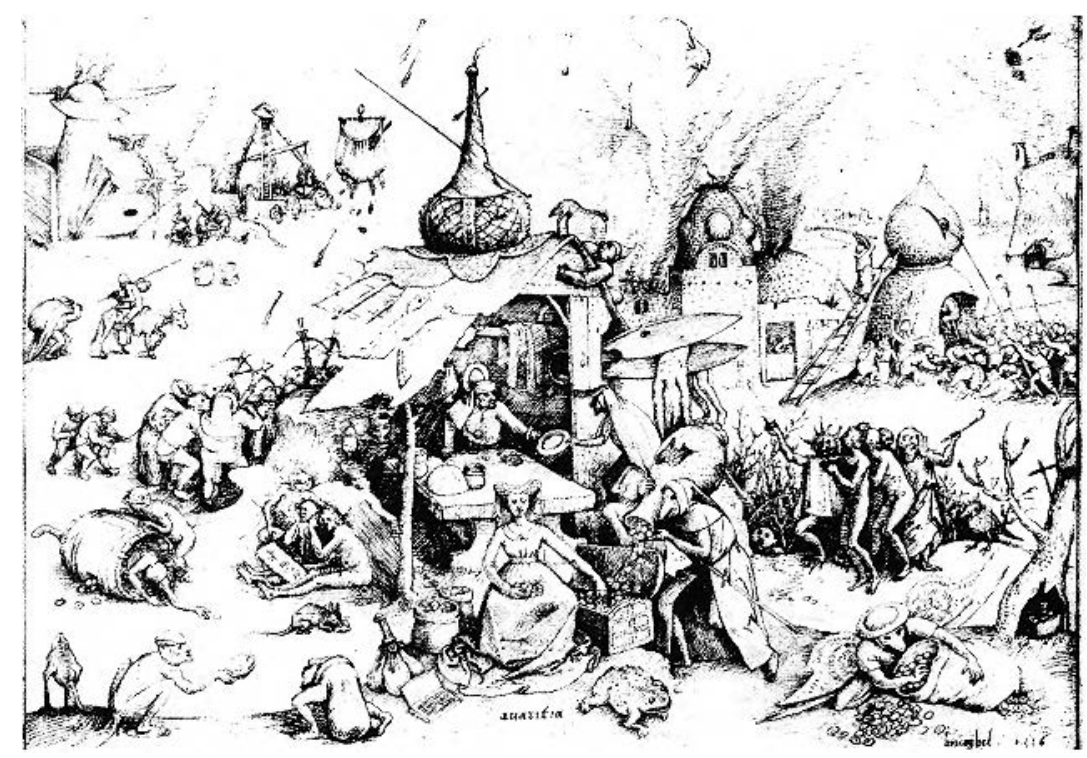

figure 1. Pieter Breughel le Vieux, l'Avarice. Londres, British Museum (Photo : Ludwig Münz, The Drawings of Breughel, Londres, Phaidon Press, $\left.19^{61}, \mathrm{pl} .127\right)$.

L'art baroque et de la Renaissance w. McAI.IISTtR JoHnson, Université de Toronto, président de séance

Dans une communication intitulée How Love Became Avarice; The Transformation of an Image, Patricia Vervoort de l'Université Lakehead suggéra que pour illustrer l'Avarice (Fig. 1) dans sa série des Sept péchés capitaux, Breughel s'est inspiré d'unc gravure du milieu du $\mathrm{xv}^{\mathfrak{e}}$ siècle par le Maître des Jardins d'amour. Il se conformait ainsi à une pratique fréquente dans les gravures du $\mathrm{XV}^{\mathrm{C}}$ et du $\mathrm{XvI}^{\mathrm{c}}$ siècle qui idéalisent l'amour aussi bien qu'elles le représentent comme une passion folle.

Examinant Le Imagini de $i$ dei de gli antichi de Vincenzo Cartari, un ouvrage du xvi" siècle apparemment conçu pour fournir des modèles aux artistes, Madeleine Lennon de l'Université de Western Ontario a surtout fait ressortir les relations qui y sont établies entre les dieux paiens et l'existence terrestre de l'homme.

Michèle Métraux de l'Université York a étudié le contexte historique qui entoure la construction de l'église carmélite de San Martino ai Monti et a analysé l'iconographie de deux de ses fresques, qui représentent l'intérieur de Saint-Jean-de-Latran et l'intérieur de la première église de Saint-Pierre, pour souligner leur importance dans le renouveau d'intérêt pour l'ère paléochrétienne qu'a connu la Rome du xvir" siècle.

Dans Trois "bodegones" inédits au Musée d'Evora, Portugal, Luis de Moura Sobral de l'Université de Montréal a soulevé certains problèmes d'ordre stylistique et iconographique que causc l'attribution de ces bodegones à Josefa de Obidos (v. 1630-1684).
Les historiens de l'art canadiens en Italie I.U B A FLEEN, Collège Erindale, Université de Toronto, présidente de séance

L'originalité de plusieurs thèmes qu'illustrent les fresques des catacombes de San Valentino à Rome confère une grande importance au problème de lcur datation. La récente publication de Per Jonas Nordhagen sur les peintures de Santa Maria Antiqua dans le Forum romain suggère à John Osborne de l'Université de Victoria que les fresques de San Valentino, qui présentent des ressemblances stylistiques avec ces dernières, pourraient elles aussi être du début du viII" siècle.

Martin Kilmer de l'Université d'Ottawa a présenté un relief illustrant l'Humiliation d'Hector qu'une équipe d'archéologues canadiens a découvert à Carthage en 1978 . Des ressemblances stylistiques avec des monuments de l'époque de la Tétrarchie conduisent Kilmer à soulever la possibilité d'une influence nord-africaine sur l'art de la Rome de Constantin.

David McTavish de l'Université Queen's a parlé de Giuseppe Salviati. Formé à Rome au début du Xvi ${ }^{\mathrm{e}}$ siècle, Salviati fit carrière principalement à Venise où son art acquit des caractéristiques vénitiennes en plus de refléter le passage des idéaux du Maniérisme à ceux de la Contre-Réforme.

L'art vénitien, mais du xiII" siècle cette fois, a été aussi le sujet de la communication de Luba Eleen. Elle a fait ressortir des rapports entre les scènes des Évangiles des Nouveaux Testaments de Vérone et les mosaïques de Saint-Marc à Venise. Des ressemblances iconographiques et stylistiques font croire qu'un artiste formé à Venise se soit déplacé à Vérone autour de l'an 1200. 


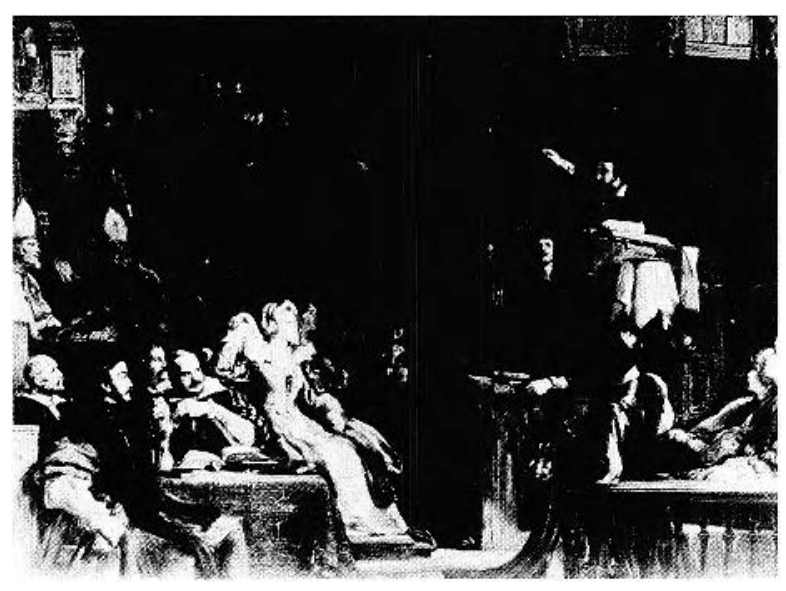

figure 2. David Wilkie, La Prédication de Knox, 1832.

Une interprétation sociale de l'art SERGE GUILBAUT, Université de la Colombie-Britannique, président de séance

Leonard Folgarait du California Institute of the Arts a exposé les facteurs qui expliquent pourquoi la gigantesque murale de l'artiste mexicain David Alfaro Siqueiros, la Marche de l'humanité sur la terre et vers le cosmos, diffère de ses ccuvres antérieures par le contenu et par le style. Communiste et prisonnier politique, Siqueiros a peint cette murale à la demande d'un homme d'affaires d'extrême-droite immédiatement après sa sortie de prison en 1964 . Une déformation complexe des codes apparents de signification conduit à exprimer l'idéologie du nouvel État capitaliste mexicain.

Exposée à la Royal Academy en 18.32 , la Prédication de Knox (Fig. 2) par David Wilkie fut immédiatement acclamée par les critiques qui écrivaient dans les journaux et revues de l'establishment. Toutefois Bridget Elliott de l'Université de la Colombie-Britannique a montré que Whigs et Tories ont retenu des aspects différents du sermon de Knox et qu'ils s'en sont servi pour appuyer leurs positions opposées sur la réforme parlementairc qu'ils allaient voter un mois plus tard.

Elizabeth Kahn Baldewicz de l'Université St. Lawrence, Canton (N.Y.), a soutenu que la Première Guerre mondiale a exercé un impact profond sur la vision artistique et les théories de l'art cn France durant cette période, même si la littérature sur l'art du $x^{\mathfrak{c}}$ siècle écarte complètement cette question.

David H. Solkin de l'Université de la ColombieBritannique proposa une interprétation du White Monk de Richard Wilson comme un concordia discors. Ce concept de l'univers reposant sur l'union harmonieuse d'éléments divers $\mathrm{a}, \mathrm{au}$ xvIII ${ }^{\mathrm{C}}$ siècle, été exploité par l'aristocratie et la petite noblesse pour consolider leur hégémonie, et cela expliquerait le succès considérable du tableau dc Wilson qu'il a lui-même produit en de nombreuses versions.
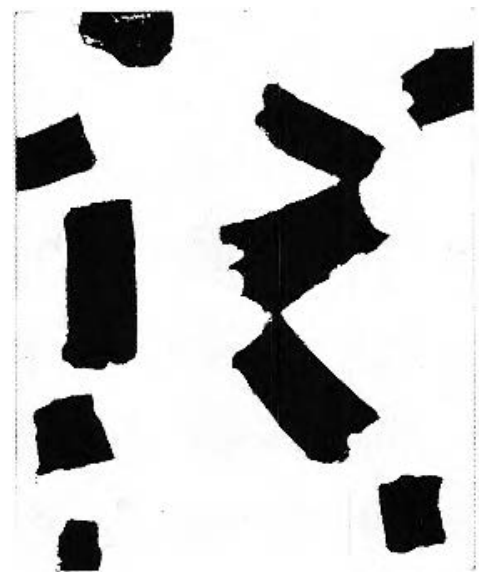

Figure 3. Paul-Émile Borduas, $3+3+4,1956$. Montréal, Musće d'art contemporain (Photo : Musée d'art contemporain).

L'art contemporain au Québec NICOLE DUBREUILBLONDin, Université de Montréal, présidente de séance

La session sur l'art contemporain au Québec s'est caractérisée par la diversité des approches, à l'exclusion de la méthode purement historique favorisée ailleurs. Dans l'exposé de Fernande Saint-Martin de l'Université du Québec à Montréal, une sémiologie topologique des arts visuels permit une relecture d'une ceuvre abstraite de Borduas (Fig. 3). En plus de raffiner les mécanismes de l'analyse formelle, la démarche révéla une structure inédite de l'espace pictural dont les significations complexes outrepassent les références paysagiques déjà repérées dans l'xuuvre.

Optant pour une approche sociale de l'histoire de l'art, Judith Ince de l'Université de la ColombieBritannique examina les concepts de liberté proposés par les Automatistes et par les artistes de Prisme d'Ycux. Au-delà d'une simple rivalité artistique, elle repéra dans l'idéologie politique de l'époque les fondements des stratégies des deux clans.

Intéressée elle aussi par les Automatistes et par une approche sociologique, Lise Lamarche de l'Université de Montréal proposa une réflexion sur la notion de groupe, un concept que l'histoire de l'art manipule constamment et dont les frontières mouvantes découlent d'a priori rarement avoués.

Une analyse de dessins de jeunes peintres québécois permit à France Gascon du Musée d'art contemporain de Montréal d'envisager les particularités de ce médium qui servent néanmoins de révélateur à la peinture elle-même et qui en fondent une pratique renouvelée.

Combinant les apports de la psychanalyse à ceux de la linguistique, René Payant de l'Université de Montréal se pencha sur l'autoportrait pour montrer que la mise en scène de l'image de soi renverse la perspective figurative et pose obliquement la question de l'identité du sujet peignant, du signe pictural et de la représentation. 
L'art documentaire au Canada RAYMOND VÉZINA, Archives publiques du Canada, président de séance

En plus d'un professeur d'histoire de l'art, cette séance réunissait les conservateurs d'importantes collections en iconographie canadienne qui ont chacun présenté leur collection.

La collection de la section canadienne du Royal Ontario Museum dont a parlé Mary Allodi se compose de portraits de personnes associées à l'histoire du Canada, de vues topographiques de chaque région du Canada, ainsi que de vues illustrant des événements importants de l'histoire canadienne aussi bien que des scènes de la vie courante. Mary Allodi suggéra deux types de catalogues: le type inventaire (Canadian Watercolours and Drawings in the Royal Ontario Museum, 1974); le type étude, comme le catalogue de la récente exposition d'estampes imprimées au Canada avant 1850.

Les premières collections de la Fondation Glenbow dont a parlé Patricia Ainslie ont été constituées par Eric Harvie entre 1954 et 1966 , date à laquelle il en fit don à la province d'Alberta. La collection d'art se compose d'environ 30 ooo ceuvres qui illustrent surtout l'histoire de l'Ouest canadien. La collection possède en outre un certain nombre d'œuvres provenant d'autres régions et même d'autres pays. Environ $95 \%$ de la collection est inventorié et photographié. Les chercheurs ont accès à toute cette information.

John Crosthwait a présenté la Canadian Historical Picture Collection de la Metropolitan Toronto Library. Celle-ci renferme environ 100 tableaux, 3 7oo aquarel- les et dessins, 10 ooo dessins à la plume et 200 gravures. Ces ceuvres illustrent principalement des scènes de Toronto et des portraits de Torontois depuis 1793 Deux catalogues de la collection ont été publiés : Landmarks of Canada, a Guide to the J. Ross Robertson Canadian Historical Collection, publié en 1917-1921 et à nouveau en 1967, et Toronto and Early Canada publié en 1964 .

Valerie Simpson du Musée du Nouveau-Brunswick a rappelé que ce musée a été fondé en 1934 grâce à l'initiative du docteur John Clarence Webster et de quelques autres personnes. Le deuxième étage de l'édifice actuel abrite la Canadian Pictorial History Gallery qui renferme 7 ooo œuvres collectionnées et données au musée par le docteur Webster. Un catalogue en trois tomes réunit tout le matériel de cette collection et un nouvel inventaire est en préparation.

W. McAllister Johnson de l'Université de Toronto a parlé des attentes du milieu universitaire et du grand public en matière d'art documentairc. Après avoir mentionné la manière habituelle de faire (expositions portant sur des peintres particuliers et dont l'envergure est forcément limitée, catalogues toujours inadéquats), il souligna les insuffisances du programme du Répertoire national. Il s'agit, à son avis, d'une expérience coûteuse fournissant des informations peu sûres. Il réclame plutôt des outils simples, à bon marché : listes sommaires, guides de collections, catalogues mis à jour régulièrement. Son expérience de l'index du Canadian Illustrated News le convainc que de tels projets sont possibles malgré les nombreux problèmes qui surgissent inévitablement. 